Bundesgesundheitsbl 2010 $\cdot 53: 84$

DOI 10.1007/s00103-009-1026-y

๑) Springer Medizin Verlag 2010

Mitteilungen des Arbeitskreises Blut des Bundesministeriums für Gesundheit

\title{
Aufrechterhaltung der Blutversorgung bei einer Influenza-Pandemie
}

\section{Am 30.11.2009 wurde folgendes Votum (V 40) verabschiedet:}

4. Impfung des für die Aufrechterhaltung der Gewinnung von Blut und Blutbestandteilen unverzichtbaren Personals, sobald ein Impfstoff zur Verfügung steht.

5. Organisatorische und Hygiene-Maßnahmen zur Vermeidung der Weiterverbreitung von Infektionen bei den Spendeterminen.

6. Information spendewilliger Personen über diese erweiterten organisatorischen und Hygiene-Maßnahmen zur Reduzierung der Angst vor Ansteckung mit Influenza bei einem Spendetermin.

7. Organisatorische Maßnahmen für die Kooperation der Blutspendeeinrichtungen in allen Belangen, die zur Aufrechterhaltung der Blutversorgung beitragen.

8. Organisatorische Maßnahmen in den Einrichtungen der Krankenversorgung zur Verringerung des Verbrauchs von Blutkomponenten.

Einzelheiten zu den verschiedenen Maßnahmen sind der Stellungnahme des Arbeitskreises Blut zu entnehmen. Die Umsetzung der oben genannten Maßnahmen erfolgt eigenverantwortlich durch die Spendeeinrichtungen und Einrichtungen der Krankenversorgung, in angemessener Form an die jeweilige Situation adaptiert.

\author{
Für den Arbeitskreis Blut \\ Prof. Dr. R. Burger, Vorsitzender \\ Dr. R. Offergeld, Geschäftsführerin
}

\section{Literatur}

1. Arbeitskreis Blut: Stellungnahme zur Aufrechterhaltung der Versorgung mit Blutprodukten im Falle einer Influenza-Pandemie. Bundesgesundheitsbl Gesundheitsforsch Gesundheitsschutz, 2009; 52 (12): 1210-1223. http://www.RKl.de > Infektionsschutz > Blut/Transfusionsmedizin $>$ AK Blut $>$ Stellungnahmen

Eine englische Version der Voten des Arbeitskreises Blut ist verfügbar unter http:// www.RKI.de > English > Prevention of Infection $>$ National Advisory Committee „Blood“ 